\title{
A COMPUTAÇÃO EM NUVEM NA UNIVERSIDADE DE SÃO PAULO
}

MARCELO KNÖRICH ZUFFO

SÉRGIO TAKEO KOFUJI

ROSELI DE DEUS LOPES

ADILSON HIRA 


\section{RESUMO}

A computação em nuvem é uma tendência observada após a Internet, a partir 1995, deixar de ser exclusivamente acadêmica e passar a ser também explorada comercialmente por empresas em escala global. Nesses últimos dezoito anos, observou-se rapidamente uma enorme incorporação da Internet no cotidiano, de tal forma que, no ano de 2012, 2,5 bilhões de humanos já se encontram conectados à rede. Essa expansão foi acompanhada por uma intensa atividade de inovação tecnológica e comercial. Dentre as inovações tecnológicas destacamos a evolução da microeletrônica e a virtualização. A computação em nuvem surge como um mecanismo para o uso intensivo de recursos computacionais, e o acesso à "nuvem" passa a ser realizado por dispositivos móveis e portáteis a partir de qualquer lugar do planeta. Este artigo discute a adoção do paradigma de computação em nuvem na Universidade de São Paulo e seu potencial impacto nas atividades de ensino, pesquisa e extensão.

Palavras-chave: Internet, computação em nuvem, Internet avançada.

\section{ABSTRACT}

Cloud computing is a trend that has been observed after Internet, starting in 1995, reached beyond the academic realm and started to be also commercially exploited by companies in global scale. In the last 18 years, we can notice how Internettechnology has been rapidly and highly integrated into everyday life at such a fast pace that in the year of 2012, 2.5 billion people were already connected to the Web. Such expansion has been followed by an intense activity of commercial and technological innovation. Among the technological innovations we highlight the evolution of microelectronics and virtualization. Cloud computing has emerged as a mechanism that enables an intensive use of computing resources; and the "cloud" can be accessed from mobile and portable devices anywhere in the world. This article discusses the adoption of the cloud computing paradigmatthe Universityof São Paulo, and its potential impact on the activities of teaching, research, and extension.

Keywords: Internet, cloud computing, advanced Internet. 


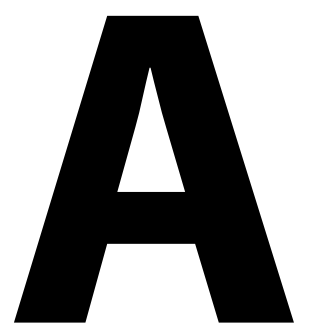

computação em nuvem é um novo paradigma da informática com foco na prestação de serviços e na gestão e governança da infraestrutura computacional. Com o advento e a adoção intensiva de microcomputadores pessoais conectados extremamente portáteis, como laptops, smartphones e tablets, novas oportunidades de entrega de serviços de informática surgiram. A disponibilidade da Internet sem fio (wireless) de forma abrangente cada vez mais tem popularizado esses microcomputadores portáteis também denominados de dispositivos inteligentes (smart devices), dispositivos conectados ou também dispositivos móveis. Neste artigo utilizaremos a denominação dispositivos inteligentes conectados (smart connected devices), pois, além de serem mais sofisticados que um simples aparelho de telefonia móvel, podem estar permanentemente conectados à Internet e são capazes de executar aplicações sofisticadas e "inteligentes" quando conectados à nuvem.

A vasta e crescente massificação dos dispositivos conectados inteligentes impulsionou o modelo Byod (bring your own device). Atualmente o usuário comum tem a sua disposição o próprio dispositivo conectado, que tem sido a principal plataforma de acesso a serviços, inclusive aqueles relacionados com a sua atividade profissional. Considerando o uso essencialmente individual e a capacidade de processamento dos dispositivos inteligentes conectados, estes passaram a incorporar muitos dos recursos do computador pessoal de mesa (desktop personal computer - PC). Para se ter uma ideia desse fenômeno, espera-se, em 2013, vender no mundo 190 milhões de tablets, que, em poucos anos, vão superar a quantidade dos computadores pessoais de mesa. Segundo a Anatel (Agência Nacional de Telecomunicações), em 2012 o Brasil chegou a 263 milhões de linhas conectadas à infraestrutura de telefonia celular (Anatel, 2013), fenômeno que tem contribuído também para a migração dos serviços para a nuvem e a diminuição do uso dos PCs.

A possibilidade de os dispositivos inteligentes conectados estarem permanentemente ligados à Internet e, portanto, a uma estrutura computacional ilimitada tem mudado o enfoque das corporações considerando as novas formas como as empresas desenvolvem e entregam os seus serviços de informática. Atualmente, há uma forte tendência das corporações públicas e privadas, empresas e governos, de confinar a infraestrutura de informática em grandes data centers públicos e privados e concentrar-se especificamente no desenvolvimento e oferta de serviços. No contexto da computação em nuvem, deve-se considerar a definição de público e privado - o termo "público" refere-se a serviços e

\section{MARCELO} KNÖRICH ZUFFO é professor titular do Departamento de Engenharia de Sistemas Eletrônicos da Escola Politécnica da USP, pesquisador do Laboratório de Sistemas Integráveis (LSI) e coordenador do Centro Interdisciplinar em Tecnologias Interativas (Citi), ambos da USP.

\section{SÉRGIO TAKEO}

KOFUJ é professor do Departamento de Engenharia de Sistemas Eletrônicos da Escola Politécnica da USP, pesquisador do LSI e do Citi.

\section{ROSELI DE DEUS}

LOPES é professora associada do

Departamento de Engenharia de Sistemas Eletrônicos da Escola Politécnica da USP, pesquisadora do LSI e vice-coordenadora do Citi.

\section{ADILSON HIRA} gerente do Núcleo de Telemedicina da área de Meios Eletrônicos Interativos do LSI/USP. 
infraestruturas compartilhados por várias entidades e indivíduos, enquanto o termo "privado" refere-se ao uso exclusivo de uma entidade e indivíduos.

Neste artigo vamos explorar esse cenário apresentado da computação em nuvem e seu contexto na Universidade de São Paulo. Na primeira parte apresentamos as características essenciais da computação em nuvem; na segunda, discutimos o paradigma de "tudo como serviço" e suas eventuais implicações em grandes entidades, como é o caso da Universidade de São Paulo.

\section{COMPUTAÇÃO EM NUVEM}

A computação em nuvem é uma tendência observada após a transição comercial da Internet realizada em 1995. Naquele instante, a Internet já estava consolidada como uma rede mundial de comunicação de dados multimídia, conectando a maioria das universidades e centros de pesquisa do mundo. Alguns anos antes, o conceito da WWW (world wide web) tinha sido criado, permitindo uma ampla expansão e aceitação da Internet por usuários leigos em computação. A world wide web tinha, como intenção, facilitar a organização e a navegação dos dados por usuário comum na Internet, introduzindo conceitos revolucionários, como a navegação hipermídia, e tecnologias, como o sistema de endereçamento URL (uniform resource locator), o protocolo HTTP (hypertext transfer prococol) e a linguagem HTML (hypertext markup language), tecnologias que foram a base para o estabelecimento da computação em nuvem.

Naquele instante do surgimento da WWW, o termo "nuvem" começou a ser consagrado para designar o conjunto de equipamentos que compõem a infraestrutura da rede Internet, como computadores, comutadores (switches), roteadores e impressoras. A partir de então, alguns serviços começam a ser prestados através dos navegadores (browsers). Ao longo da década de 1990, surgiram várias iniciativas de compartilhamento de dados, computação e instrumentos na rede WWW, centenas de projetos de pesquisa, como o sequenciamento do genoma humano, Xylella (Fapesp, 2013), instrumentação virtual, entre outros.

Com o avanço da ciência e da engenharia na área de computação, impulsionadas também pela própria WWW, as técnicas de compartilhamento de recursos computacionais evoluíram rapidamente, consolidando a tecnologia de virtualização, que compreende vários mecanismos de dissociação das aplicações computacionais de seus recursos físicos, dentre eles as máquinas virtuais, que são simulações dos próprios computadores. Com a maturidade dessa tecnologia, praticamente todos os elementos da infraestrutura física e todos os serviços podem ser virtualizados, ou seja, as antigas infraestruturas de informática, como CPDs (centrais de processamento de dados), computadores de mesa, servidores e centrais telefônicas podem ser desmaterializadas através de recursos de virtualização e disponibilizadas na nuvem. A desmaterialização da infraestrutura tradicional de informática traz inúmeras vantagens relacionadas com a gestão física de patrimônio, gerenciamento da obsolescência, manutenção e acesso à infraestrutura.

\section{Características essenciais da computação em nuvem}

A computação em nuvem é um conceito que continua sofrendo inúmeras evoluções na sua definição devido à forte atividade de inovação em torno dele. O National Institute of Standards and Technology (Nist), nos Estados Unidos, apresenta a seguinte definição:

"A computação em nuvem é um modelo para permitir, convenientemente, o acesso à rede sob demanda a um conjunto compartilhado de recursos computacionais configuráveis (por exemplo, redes, servidores, armazenamento, aplicativos e serviços) que podem ser rapidamente provisionados e liberados com mínimo esforço de gestão ou interação com o prestador de serviços" (Nist, 2013).

Considerando essa definição, o Nist elenca cinco características essenciais da com- 
putação em nuvem que serão apresentadas a seguir e discutidas no contexto da Universidade de São Paulo.

\section{Serviço sob demanda}

Nesse contexto, o usuário ou consumidor pode solicitar de forma unilateral recursos computacionais de que precisa automaticamente da nuvem, sem a necessidade de nenhuma intermediação humana. Esses recursos computacionais podem ser capacidade computacional, armazenamento, impressão ou recursos virtualizados, como servidores, computadores desktop e redes virtuais.

Essa característica da computação em nuvem oferece grande conveniência aos usuários, que podem usar os serviços a partir de qualquer lugar. $\mathrm{O}$ agrupamento dos recursos computacionais traz enormes vantagens em gestão do patrimônio, otimização de gastos e diminuição de esforço humano em atividades de manutenção de equipamentos físicos.

\section{Amplo acesso à rede}

Todos os recursos oferecidos pela nuvem devem ser acessados necessariamente pela rede, através de mecanismos padronizados que garantam interoperabilidade, não repúdio, consistência, segurança, disponibilidade e robustez dos serviços prestados. Esse acesso deve considerar uma ampla diversidade de dispositivos de acesso no contexto do Byod.

Cabe ressaltar que a existência de uma rede com essas características é condição essencial para a computação em nuvem. A interoperabilidade é a garantia de que qualquer dispositivo pode ser interligado à rede e de que vai funcionar. $\mathrm{O}$ não repúdio está associado à identificação do usuário, garantindo, de forma legal, o reconhecimento de sua existência íntegra e seu acesso à nuvem.

\section{Agrupamento de recursos}

Na computação em nuvem, os recursos computacionais físicos e virtuais são agrupados para servir simultaneamente a múltiplos usuários. Esses recursos podem ser dinamicamente alocados de acordo com as necessidades de desempenho, escala e demanda. Uma característica associada ao agrupamento de recursos é a virtualização e o fato de existir uma independência de localidade, ou seja, na nuvem o usuário não tem a percepção de onde o seu serviço ou recurso virtual está sendo fisicamente executado. No caso de nuvens públicas ofertadas por grandes empresas multinacionais, os serviços podem ser executados em praticamente qualquer lugar do mundo.

\section{Elasticidade rápida}

A elasticidade rápida é uma característica importante da computação em nuvem, pois os recursos computacionais podem ser provisionados automaticamente e de forma muito rápida. $\mathrm{O}$ usuário final tem uma percepção de recursos ilimitados que podem crescer rapidamente, mas que obviamente devem e precisam ser associados a custos para que sejam utilizados de forma adequada pelos usuários. Ao mesmo tempo em que os recursos podem ser rapidamente disponibilizados, também podem ser retirados rapidamente e colocados à disposição de outros usuários. Esta é a situação típica encontrada no meio científico, onde eventuais experimentos computacionais devem ser utilizados com grande capacidade de processamento por curtos espaços de tempo.

\section{Serviço medido}

A última característica importante da computação em nuvem é a medição dos serviços prestados em tempo real. Essa monitoração pode ser em diversos níveis de abstração, como bytes armazenados, bits por segundo transmitidos, MIPs (milhões de instruções de processamento por segundo), número de máquinas virtuais alocadas, entre outros. A característica de serviço medido é fundamental para a avaliação de uso da infraestrutura da computação em nuvem e para a gestão e a alocação apropriadas de seus recursos à comunidade de usuários. 


\section{Governança e gestão}

Outra forma de abordar o paradigma da computação em nuvem é do ponto de vista de governança e gestão. Para os gestores da área de tecnologia de informação e também os de grandes organizações voltadas à geração de conhecimento, como é o caso da Universidade de São Paulo, a computação em nuvem é uma excelente oportunidade de organizar e otimizar recursos e qualidade de serviços, desde que haja observância estrita de suas características essenciais.

Além das características essenciais preconizadas pelo Nist, a computação em nuvem apresenta a perspectiva da economia de escala, tanto para pequenas como para grandes organizações. Supondo a opção por uma estrutura de computação em nuvem privada, alguns princípios devem nortear a implementação dessa infraestrutura, como a padronização de processos e equipamentos, a replicação de processos e recursos e a sustentabilidade.

Segundo o Anuário Estatístico da USP publicado em 2012, oficialmente a universidade possui aproximadamente $55 \mathrm{mil} \mathrm{com-}$ putadores (USP, 2012). Esse parque instalado é significativo. Considerando a relação docente por computador, temos uma relação aproximada de 10 para 1 , ou 5 para 1 , considerando a relação funcionário por computador, ou 1 para 2, considerando a relação aluno por computador. Esses números posicionam a USP como uma das organizações mais informatizadas do Brasil, levando em conta também a dimensão da universidade.

Claramente observa-se uma dificuldade muito grande em manter esse parque de computadores operacional e atualizado, considerando os ciclos de obsolescência da informática, que são normalmente de 18 a 24 meses, bem como os ciclos de aquisição de bens e consumíveis no âmbito da legislação de compras públicas, no caso, a Lei de Licitações 8.666. Alia-se, a esse contexto, a necessidade de equipamentos cada vez mais sofisticados de computação para a realização de pesquisas avançadas nos mais diversos e abrangentes campos da ciência.

\section{Tudo como serviço}

A prestação de serviços convenientes on-line, sob demanda, em qualquer lugar e com oferta de recursos ilimitados, passa a ser o grande foco de atenção da computação em nuvem. Esta seção discute algumas questões relacionadas a esse modelo de serviço e introduz o conceito de "servitização".

A servitização é definida como a área de estudo relacionada com a gestão da operação de serviços. Trata-se de um termo também associado à inovação na oferta de serviços por organizações que tradicionalmente ofertavam serviços ou produtos no contexto da sociedade industrial e passaram a se reinventar prestando serviços na sociedade do conhecimento. Talvez este seja o desafio de grandes organizações, como a Universidade de São Paulo, quando confrontadas ao paradigma da computação em nuvem.

A computação em nuvem cria um jargão específico para o contexto da servitização. Especificamente, o Nist estabelece três camadas de serviços: software como serviço (software as a service - SaaS), plataforma como serviço (platform as a service - PaaS), infraestrutura como serviço (infrastructure as a service -IaaS). A estruturação dessas camadas é apresentada na Figura 1. Quanto mais no topo da pirâmide de serviços, mais próximos esses serviços estão dos usuários; quanto mais na base da pirâmide, mais próximos estão da infraestrutura física.

Serviços tipo software como serviço ou SaaS são aqueles baseados em aplicações acessíveis por diversos dispositivos conectados, desde dispositivos móveis até dispositivos de mesa, como equipamentos thin client. Normalmente essas aplicações são executadas a partir de navegadores (browsers). Nesse caso, o usuário não tem controle ou gerenciamento sobre a infraestrutura que suporta essas aplicações.

Serviços em nuvem tipo plataforma como serviço ou PaaS são aqueles baseados em plataformas de desenvolvimento, como ferramentas, ambientes e linguagems de programação. Novamente o usuário não tem acesso 


\section{FIGURA 1}

\section{HIERARQUIA DE SERVIÇOS DE ACORDO COM A PROPOSTA DO NIST}

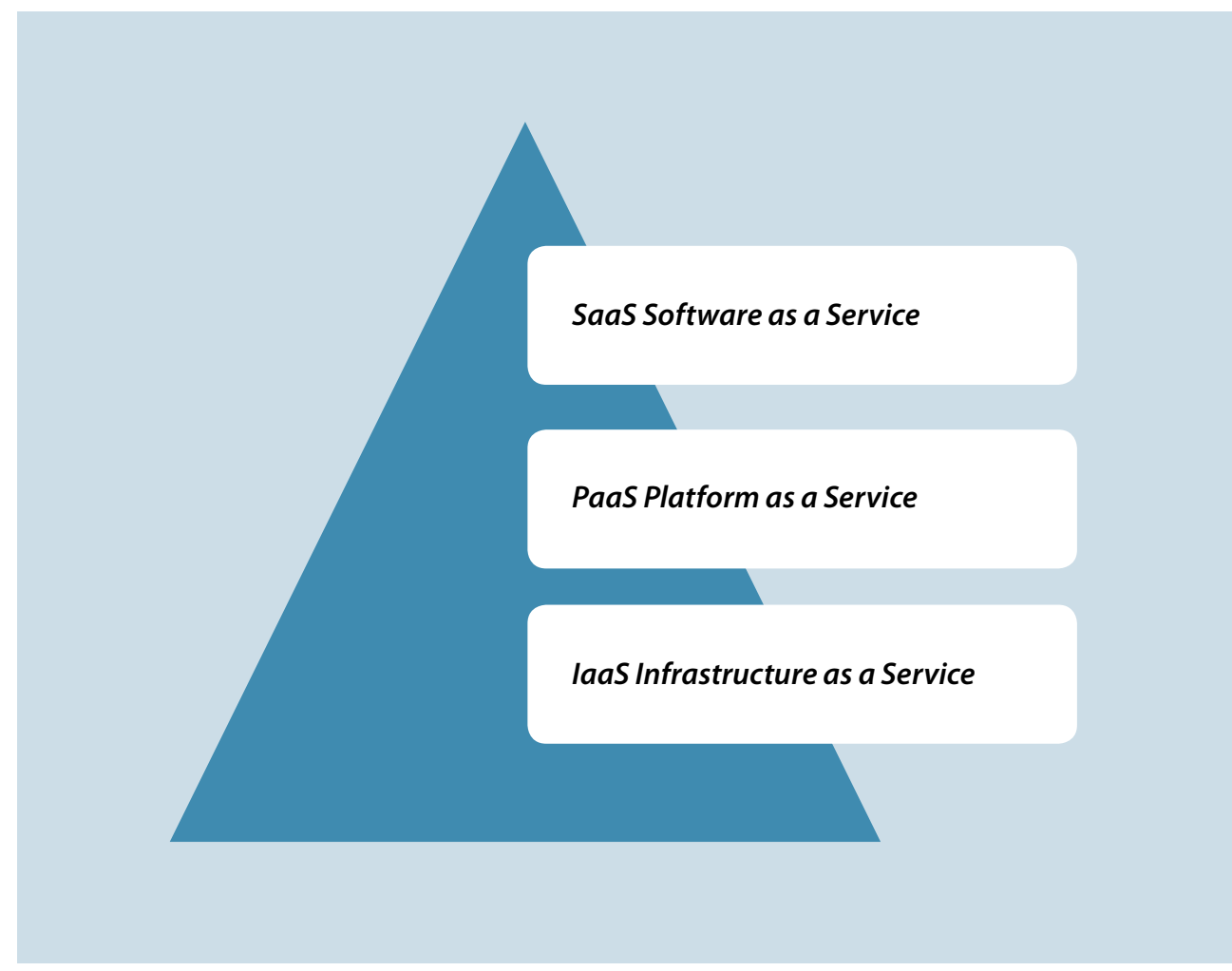

ao gerenciamento e ao controle das camadas de infraestrutura, como rede, servidores e sistema operacional. No caso, o usuário tem controle sobre as aplicações que desenvolveu sobre o ambiente PaaS.

Finalmente, serviços em nuvem tipo infraestrutura como serviço ou IaaS são aqueles baseados em recursos computacionais básicos, como processamento, rede, armazenamento e máquinas virtuais, que podem ser solicitados pelo usuário para a execução de qualquer ambiente de software. Normalmente o domínio de serviços IaaS é onde o conceito de virtualização é mais presente. Nele, o usuário tem controle sobre os parâmetros do ambiente virtualizado, mas não possui acesso e/ou gerenciamento aos recursos físicos.

\section{COMPUTAÇÃO EM NUVEM E SUSTENTABILIDADE}

A Universidade de São Paulo é considerada uma large university segundo diversos sistemas de classificação de universidades, sendo geograficamente distribuída em diversos campi, tanto na cidade como no estado de São Paulo. A instalação, a operação, a manutenção e, principalmente, a contínua atualização da infraestrutura informática, incluindo servidores e rede de comunicação de dados, em uma universidade desse porte, representam um grande desafio, principalmente tendo-se em conta a rápida obsolescência de tecnologias e equipamentos envolvidos. Não menos importante é a questão da 
sustentabilidade, notadamente com relação ao consumo de energia elétrica e reciclagem. É o que conhecemos hoje pelo termo "TI verde" (Murugesan, 2008).

A contínua atualização dos recursos de TI traz à tona diversos problemas relacionados com o conceito de TI verde:

- o descarte de lixo eletrônico - produtos eletrônicos obsoletos;

- o uso de tecnologia de alta eficiência visando à redução do consumo total de energia em computação e comunicação;

- a redução do impacto ambiental do sistema de refrigeração associado a ambientes climatizados;

- a redução do impacto ambiental relativo ao sistema de energia secundária (no-breaks e geradores).

Uma discussão da questão da sustentabilidade passa pelo conhecimento dos diversos perfis de uso dos recursos de TI dentro de uma universidade. A USP tem os seguintes domínios de necessidade em termos de computação em nuvem:

- domínio educacional: relaciona-se ao apoio para alunos e professores em atividades de graduação e pós-graduação. Inclui portais educacionais para apoio às disciplinas, ferramentas de apoio ao ensino presencial e a distância, ferramentas diversas. O sistema de comunicação deve permitir o acesso às ferramentas computacionais dentro e fora das instalações físicas (salas de aula, laboratórios, anfiteatros), de forma ubíqua, por dispositivos. A disponibilização de conteúdo de disciplinas de graduação e pós-graduação de forma livre na Internet é uma tendência, que a USP também segue;

- domínio científico: relaciona-se ao apoio aos docentes e pesquisadores nas atividades de pesquisa e desenvolvimento voltadas à geração de conhecimento. Inclui infraestruturas de computação e armazenamento de alto desempenho, programas científicos, infraestruturas de trabalho colaborativo e sistemas de visualização científica avança- da. Esse domínio ainda inclui bibliotecas e outros acervos. Apesar da tendência do acesso a livros e periódicos pela Internet, as bibliotecas da universidade ainda são uma importante fonte de informação, tanto para o ensino, como também para a pesquisa. Cada vez mais a informação é gerada, na universidade, na forma de teses e dissertações digitais. Cabe às bibliotecas a organização dos repositórios de informações bibliográficas para o acesso dos docentes, alunos, pesquisadores e sociedade e, também, a organização e divulgação dos conteúdos científicos produzidos pela universidade (por exemplo, teses de doutorado, dissertações de mestrado, trabalhos de conclusão de curso, relatórios internos e artigos acadêmicos). Finalmente, temos os diversos museus mantidos pela universidade, os quais, com a digitalização das obras, têm a cada dia uma base de dados maior e com acesso mais interativo;

- domínio administrativo: contempla todo o universo de aplicações e sistemas administrativos da universidade, como folha de pagamento, matrícula, e-mail de docentes, funcionários, alunos e, eventualmente, ex-alunos, emissão de diplomas, gestão de processos administrativos, gestão da documentação eletrônica, compras, dentre inúmeros outros. Cabe salientar que esse domínio pode incluir também os hospitais associados à USP, nos quais os fluxos de trabalhos voltados ao atendimento à saúde estão sendo totalmente informatizados. Neles, os prontuários de pacientes poderiam se tornar totalmente digitais, para o que seria necessário o uso intensivo do certificado digital, descartando a necessidade de manipulação e armazenamento de grande volume físico de papel e filme.

Pode-se perceber que, em todos os domínios apresentados, a disponibilização de uma infraestrutura de computação e comunicação, com acesso transparente dentro da universidade, capaz de armazenar dados, fornecer acesso a programas diversos e disponibilizar uma infraestrutura de computação 
de alto desempenho, é altamente vantajosa. O oferecimento de computação de alto desempenho em nuvens ainda é um tópico em evolução, uma vez que nem todas as infraestruturas de nuvem suportam computação de alto desempenho (por exemplo, podemos citar os seguintes pontos: suporte e passagem de mensagens eficientes, hardware de rede de comunicação de alta velocidade, sistema de gerenciamento e escalonamento de tarefas, etc.), e nem todas as aplicações de alto desempenho são facilmente portadas para nuvens (um exemplo é a dificuldade de porte de aplicações de física de altas energias para uma nuvem qualquer, independentemente do sistema de virtualização) (Ekanayake \& Fox, 2010). A infraestrutura de computação, no modelo de computação em nuvem, é acessada pelos usuários através de uma infraestrutura pervasiva de comunicação, que inclui acesso cabeado e acesso sem fio WiFi e também via rádio celular.

Instituições como a Universidade de São Paulo, ao adotarem a computação em nuvem privada (Armbrust et al., 2010), obtêm vantagens advindas da consolidação de servidores, da virtualização, da racionalização de recursos, evitando duplicidade de servidores, podendo também se beneficiar em termos de sustentabilidade, notadamente em relação (mas não se limitando) a questões de energia Dessa forma, pode-se perceber que a comunidade ligada à universidade se beneficia, com maior ou menor intensidade, do uso de computação em nuvem, obtendo acesso ubíquo às facilidades de TI através de computadores de mesa e thin clients, notebooks e dispositivos portáteis, como os tablets e smartphones. Dependendo das necessidades dos usuários, há maior demanda em termos de processamento nos servidores da nuvem ou em termos do uso de banda e das capacidades de especificação dos níveis de qualidade de serviço na infraestrutura de comunicação na rede.

Uma vantagem importante do modelo de computação em nuvem é permitir que a infraestrutura computacional, localizada em data centers, possa ser facilmente atualizada de forma a utilizar tecnologias modernas mais sustentáveis, principalmente no quesito de energia. Importante notar, todavia, que a simples consolidação de servidores através de virtualização na infraestrutura computacional não se traduz automaticamente em redução de energia. $\mathrm{O}$ uso de computadores com eficiência energética (e térmica), tanto no lado da nuvem como também dos usuários (por exemplo, através do incentivo de uso de thin clients e ultrabooks), é fundamental para a obtenção de bons resultados.

À medida que os diversos servidores da universidade, nem sempre eficientes em termos energéticos, vão sendo migrados e consolidados nos data centers da nuvem, temos um potencial ganho de energia, seja pela utilização de servidores com tecnologia mais atual, seja pela redução do impacto ambiental decorrente da otimização da arquitetura física do ambiente, onde servidores e seus periféricos são instalados, otimizando o sistema de refrigeração e minimizando as perdas de energia decorrentes de, por exemplo, necessidade de iluminação constante, redução global do cabeamento, com consequente minimização de perdas elétricas, simplificação dos sistemas de segurança, dentre inúmeras outras vantagens.

Para acessar a nuvem (infraestrutura, plataforma ou software), computadores mais leves, como thin clients, podem ser utilizados. Evidentemente, assume-se, nesse cenário, uma infraestrutura de comunicação bem dimensionada que garanta um acesso de qualidade na ponta. Particularmente no caso da USP, há também o problema de prover uma infraestrutura de comunicação entre os diversos campi.

Em relação à atualização da infraestrutura computacional da nuvem, para resolver o problema de descarte de produtos eletrônicos, pode-se cogitar em um modelo de atualização com substituição. Já em relação aos computadores de acesso à nuvem, o problema é menos crítico do que no modelo sem nuvem, pois não há necessidade de substituição dos computadores de acesso para a obtenção de maior desempenho. Todavia, computadores de acesso mais eficientes energeticamente 
são sempre uma política interessante visando à redução do gasto de energia, não só devido ao computador, como também ao sistema de refrigeração e no-breaks.

\section{CONCLUSÃO}

A computação em nuvem tem sido objeto de intenso debate no meio técnico e acadêmico, não só pelo seu contexto de forte tendência observada na área de computação, como também pelos impactos gerados pela sua intensa adoção por organizações no mundo todo.

No âmbito da Universidade de São Paulo, discussões e atividades relacionadas com a computação em nuvem vêm sendo realizadas nos últimos anos, inclusive com o desenvolvimento do projeto Nuvem USP, conduzido pela Superintendência de Tecnologia da Informação, em parceria com a Vice-reitoria Executiva de Administração, ambos órgãos centrais e complementares vinculados à Reitoria da universidade.

A adoção consistente, no longo prazo, desse novo paradigma pode modificar e aprimorar profundamente os mecanismos de geração de conhecimento na USP e de disponibilização, na sociedade, de formas mais abrangentes e democráticas, além das fronteiras geográficas e culturais do Brasil. A da digitalização intensiva de todo o acervo de conhecimento da universidade, aliada às novas ferramentas de computação científica para a manipulação e análise dessas informações, abre imensas possibilidades nas atividades de pesquisa e gestão na universidade.

\section{BIBLIOGRAFIA}

ANATEL - Agência Nacional de Telecomunicações. www.anatel.gov.br, 2013.

ARMBRUST, Michael; FOX, Armando; GRIFFITH, Rean; JOSEPH, Anthony D.; KATZ, Randy; KONWINSKI, Andy; LEE, Gunho; PATTERSON, David; RABKIN, Ariel; STOICA, Ion and ZAHARIA, Matei. 2010. "A View of Cloud Computing", in Commun. ACM 53, 4 (April/2010), pp. 50-8. DOI=10.1145/1721654.1721672. Disponível em: http://doi.acm. org/10.1145/1721654.1721672.

EKANAYAKE, Jaliya and FOX, Geoffrey. "High Performance Parallel Computing with Clouds and Cloud Technologies", in Lecture Notes of the Institute for Computer Sciences, Social-Informatics and Telecommunications Engineering. Volume 34, 2010, pp 20-38. DOI=10.1007/978-3-642-12636-9_2.

FAPESP- Fundação de Apoio à Pesquisa do Estado de São Paulo. http://www.bv.fapesp. br/linha-do-tempo/1206/genoma-xylella/, 2013.

MURUGESAN, San. "Harnessing Green IT: Principles and Practices", in IT Professional, vol. 10, no 1, pp. 24,33, Jan.-Feb./2008. DOI: 10.1109/MITP.2008.10.

NIST 2013. National Institute for Standards and Technology of The NIST Definition of Cloud Computing (NIST Special Publication 800-145). Disponível em: http://csrc.nist.gov. USP.Anuário Estatístico da Universidade de SãoPaulo, 2012.Disponível em:http://www.usp.br. 